\title{
O que é uma universidade tecnológica no Brasil?
}

\author{
What is a technological university in Brazil?
}

\author{
LARA, Luiz Marcelo de ${ }^{1}$ \\ PILATTI, Luiz Alberto ${ }^{2}$ \\ SANTOS, Celso B. dos ${ }^{3}$ \\ PEDROSO, Bruno ${ }^{4}$
}

\begin{abstract}
Resumo
O presente estudo tem como objetivo identificar as características distintivas do modelo universidade tecnológica no Brasil. O procedimento técnico adotado foi a pesquisa bibliográfica de natureza exploratória. Constatou-se que o relacionamento com o mercado e as particularidades nas missões universitárias produzem a ideia de universidade tecnológica. Conclui-se que o crescente distanciamento entre o ideário presente na concepção da Universidade Tecnológica Federal do Paraná (UTFPR) e a prática institucional produz um modelo distorcido para o Brasil.

Palavras-chave: universidade tecnológica, educação tecnológica, pesquisa aplicada, transferência de tecnología.

Abstract

The objective of this study was to identify the distinct characteristics of the technological university model in Brazil. The technical procedure adopted was the bibliographic research of an exploratory nature. We found that the relationship with the market and particularities around the aims of universities produce an idea of a technological university. We conclude that the growing divergence between the ideal present when Federal Technological University of Paraná (UTFPR) was conceived, and the current institutional practice produces a distorted model for Brazil.

Keywords: technological university, technical education, applied research, technology transfer.
\end{abstract}

\section{Introdução}

A primeira universidade tecnológica (UT), a Czech Technical University in Prague, foi fundada em 1707, na República Checa. Ainda no século XVIII, a ideia de UT foi difundida para a Alemanha em 1770, Turquia em 1773, Hungria em 1782, França em 1794 e Escócia em 1796. Da Europa as UTs migraram para a Ásia, apesar de que o movimento só teve escala depois da Segunda Grande Guerra. Nos Estados Unidos, local onde estão situadas algumas das universidades tecnológicas mais importantes do mundo como a California Institute of Technology e o Massachusetts Institute of Technology, a introdução do modelo ocorreu principalmente depois da segunda metade do século XIX (Pilatti e Lievore, 2018).

\footnotetext{
${ }^{1}$ Universidade Tecnológica Federal do Paraná (UTFPR). Brasil. E-mail: luizmarcelolara@hotmail.com

2 Universidade Tecnológica Federal do Paraná (UTFPR). Brasil. E-mail: lapilatti@utfpr.edu.br

${ }^{3}$ Universidade Estadual de Ponta Grossa (UEPG). Brasil. E-mail: bilynkievycz@uepg.br

${ }^{4}$ Universidade Estadual de Ponta Grossa (UEPG). Brasil. E-mail: prof.brunopedroso@gmail.com
} 
A progênie das UTs ocorreu no contexto da Revolução Industrial. O período foi permeado por mudanças sociais e tecnológicas. As mudanças produziram um cenário crescentemente complexo e com demandas até então inexistentes. Entre as demandas, a do ensino profissionalizante para suprir com mão de obra qualificada as novas indústrias (Castells, 2005; Hasanefendic, 2018).

As UTs foram fundadas com atuação predominante nas áreas de engenharia e de tecnologia ou resultaram da transformação ou aglomeração de instituições de ensino técnico-profissionalizante (Lievore e Pilatti, 2018). A inserção das UTs em diferentes espaços geográficos produziu instituições assimétricas. A assimetria produz uma imprecisão conceitual (Lievore e Pilatti, 2018; McKenna e Sutherland, 2006; Perez-Esparrells e Orduna-Malea, 2018) e, depois de três séculos de história, os contornos ainda são pouco definidos. Algo que não acontece com as universidades clássicas, detentoras de uma história iniciada no século XII.

A imprecisão conceitual é acompanhada de uma contradição. A contradição é produzida pelo antagonismo dos conceitos universidade e tecnologia que, integrados, produzem o conceito de UT. A universidade com origem no latim universitas, pode ser traduzido como universalidade, totalidade. O conceito tecnologia, originário do grego, é constituído na junção do tekne (arte, técnica, ofício) com logos (conjunto de saberes, estudo), e se caracteriza como um produto da ciência e da engenharia.

Dentro da literatura, apesar do descritor UT ser bastante utilizado, poucos estudos se debruçam de forma aprofundada na questão conceitual. Dos estudos que têm a UT como objeto dentro de uma perspectiva conceitual, predominam análises dentro do contexto institucional no espaço geográfico no qual as instituições estão inseridas (Cunnane, 2018; Doern, 2008; Du Pré, 2010; Harkin e Hazelkorn, 2015; Hatton, 2002; Hayhoe, 1989; Houghton, 2020; Kyvik, 2002; Laya, 2009; Lewis, 1991; Lievore, Pilatti e Teixeira, 2021a; McKenna e Sutherland, 2006; Mildred, 2002; Norgård e Skodvin, 2002; Pratt, 1997).

No Brasil, até em função da unicidade, os estudos, apesar de existirem, são poucos e majoritariamente no âmbito da Universidade Tecnológica Federal do Paraná (UTFPR) (Cechin, 2019; Costa e outros, 2019; Helmann, 2019; Lievore e Pilatti, 2018; Lievore, Pilatti e Teixeira, 2021a; Lievore, Pilatti e Teixeira, 2021b; Pilatti, 2017; Pilatti e Lievore, 2018).

No cenário tracejado e com foco nas três missões da universidade, o presente estudo tem como objetivo identificar as características distintivas do modelo de UT no Brasil. Para a consecução do objetivo, através de pesquisa bibliográfica, será examinada a UTFPR, ainda a única UT brasileira, na sua concepção e prática. O estudo justifica-se por sua atualidade, relevância social e incipiência no Brasil.

\section{Universidades tecnológicas em perspectiva}

Como um tipo ideal, a especialização pode ser representada em um continuum que apresenta como extremidades a especialização como meio (lato) e como fim (estrito). Enquanto representação mental, inexiste na realidade qualquer instituição que empregue a especialização integralmente como meio ou fim. No continuum, as instituições estão situadas em algum ponto, tendendo potencialmente mais para uma ou outra extremidade.

A especialização, umbilicalmente ligada com a ideia de tecnologia no conceito de UT, enquanto meio, tem ligação com o agir institucional, idealisticamente, para além das áreas do conhecimento. Com efeito, uma UT que enxerga a tecnologia como meio se aproxima da ideia de universitas. A tecnologia, assim, perde a conotação de um objetivo final para se tornar um meio curricular empregado em todas as áreas do conhecimento de forma multidisciplinar. A ideia de tecnologia como fim, na forma ideal, apresenta conexão com um modelo transdisciplinar. A especialização tem ligação com a atuação majoritária nas áreas de engenharia e de tecnologia. 
Com a perspectiva fundada na realidade latino-americana, a Red de Universidades Tecnológicas y Politécnicas da América Latina y el Caribe (RUTyP), na direção da conformação de um modelo, elegeu cinco eixos estratégicos que representam as UTs e as instituições similares existentes ao redor do mundo. As características destacadas são: inovação e empreendedorismo; pesquisa, desenvolvimento e transferência de tecnologia; parques tecnológicos e cidades do conhecimento; responsabilidade social e ambiental; e, ligação com os setores produtivos (Pilatti e Lievore, 2018; Red de Universidades Tecnológicas y Politécnicas da América Latina y El Caribe, 2017).

Du Pré (2010), para perquirir as instituições tecnológicas de ensino superior sul-africanas, identificou as características globais do modelo de UT. As características identificadas são:

a) excelência no ensino e na aprendizagem;

b) pesquisa aplicada;

c) desenvolvimento de liderança em tecnologia;

d) transferência de tecnologia e inovação;

e) parceria com a indústria (educação para o mundo do trabalho);

f) internacionalização (para benchmarking de boas e das melhores práticas).

Perez-Esparrells e Orduna-Malea (2018), ao comparar as UTs com as clássicas no Ranking THE (edição 2017), constataram um desempenho distinto. A população do estudo foram as 137 UTs ranqueadas. Na análise estatística foram identificados clusters de alto desempenho em investimentos da indústria e de baixo desempenho em ensino e pesquisa. Contrastando com as características identificadas por Du Pré (2010), forte convergência na parceria com a indústria e alguma divergência na excelência no ensino e na aprendizagem.

No contexto do Reino Unido, Lewis (1991) argumenta que, apesar de estarem inseridos em um grupo diversificado de instituições e apresentarem ethos e objetivos distintos, os institutos politécnicos possuem muitas similaridades com as universidades. Ao localizar as diferenças, Lewis (1991) aponta o perigo de procurar diferenças que podem ser mais imaginárias do que reais e de se recorrer a generalizações para se obter referências convenientes. No entanto, para o autor, as características comuns na missão dos politécnicos são:

a) o ensino é a função principal;

b) o acesso de segmentos desfavorecidos e com representação marginal na sociedade é primordial;

c) a aptidão para o ensino superior é o critério mais importante para a admissão do que os requisitos formais de admissão;

d) o forte vínculo com as comunidades locais e regionais, com o setor produtivo e com os serviços públicos;

e) os assuntos e os programas estão intimamente relacionados ao mundo do trabalho;

f) a grande importância é atribuída à validação e ao monitoramento dos padrões acadêmicos;

g) os alunos em tempo parcial constituem uma proporção significativa das matrículas;

h) uma proporção substancial das matrículas está em programas de graduação, ao invés de programas de pósgraduação.

Para Lievore e Pilatti (2018), é na simbiose entre ensino, pesquisa aplicada e transferência de tecnologia, além da proximidade com o sistema produtivo, que, em média, o modelo de UT é desenhado globalmente. Este modelo tem grande proximidade com a realidade existente na França. Apesar da falta de um conceito amplamente aceito, como o das universidades clássicas, os autores identificaram características que permitem distinguir as UTs:

a) ensino tecnológico mais prático;

b) atuação prevalente em cursos de engenharia e de tecnologia;

c) pesquisa aplicada voltada para a resolução de problemas advindos da indústria; 
d) produção e transferência de tecnologia;

e) forte vínculo com o setor produtivo;

f) ênfase na inovação e no empreendedorismo;

g) produção e utilização de tecnologia para o desenvolvimento local e regional;

h) corpo docente com experiência no setor produtivo;

i) formação de trabalhadores do conhecimento.

Na realidade concreta, a materialização das UTs é multidimensional e ocorre de forma distinta. As políticas educacionais de um país, muitas vezes determinada em função do regime político na forma de aparato legal, e a questão temporal são variáveis intervenientes no processo.

Em relação à questão temporal, é possível identificar uma cinesia importante a partir do movimento que ficou conhecido como maio de 1968. Trata-se de um movimento que teve como epicentro Paris e, além da França, atingiu vários outros países. O movimento precursor foi marcado por diversas manifestações de estudantes e de trabalhadores que reivindicavam melhores condições físicas e didáticas para as universidades e a ampliação do sistema universitário francês. Do movimento, o sistema universitário francês é remodelado com a Lei $\mathrm{n}^{\circ}$ 68-978 (France, 1968) e, em 1972, é criada a primeira universidade de tecnologia da França, a Université de Technologie de Compiègne (Cechin, Pilatti e Ramond, 2021). Decorrente do movimento que ganhou escala global, muitas instituições tecnológicas foram criadas ou transformadas na década de 1970.

Em escala planetária, principalmente na sequência do maio de 1968, têm-se dois movimentos principais, um de expansão e, outro, de redesenho.

O primeiro movimento notório é o aumento expressivo do número de UTs criadas ou transformadas. 0 movimento ultrapassou os limites da Europa, da Ásia e dos Estados Unidos, espaços nos quais estavam circunscritas a maioria das instituições tecnológicas, para, apesar de exceções notáveis, tardiamente, ganhar o globo (Hayhoe, 1989). A experiência das UTs no México é um exemplo importante neste contexto. No começo da década de 1990, o governo criou UTs para oferecer programas de dois anos destinados para egressos do ensino médio. Os programas concediam o grau de técnico superior com formação universitária (Laya, 2009).

O segundo movimento está em curso e é conformado pelo redesenho das UTs. O ponto de convergência deste movimento está na aproximação, ou na unificação, dos estatutos que distinguem instituições clássicas das tecnológicas, principalmente nas suas competências.

O credenciamento das instituições tecnológicas para a concessão de graus acadêmicos e títulos de doutorado, em vários países, é parte do redesenho. O processo já está efetivado em países como Alemanha, Holanda, Suíça, Turquia e Taiwan. Em Portugal, o processo, que não é pacífico por envolver interesses institucionais e políticos, apesar de já possuir legislação, ainda não está implantado (Lievore, Pilatti e Teixeira, 2021a, 2021b).

No Reino Unido, o sistema binário de ensino superior formado por universidades (orientadas para a pesquisa) e institutos politécnicos (congrega engenharias e ciências aplicadas e são orientados para a prática profissional) foi superado em 1992 com a designação dos politécnicos como universidades, facultando a emissão de diplomas próprios. Processo com alguma similaridade ao do Reino Unido está em curso na Irlanda e é, talvez, ao lado da incorporação da Université de Technologie de Compiègne pela Sorbonne Université, na França, em 2018 (Cechin, 2019), o exemplo mais importante em curso.

$\mathrm{Na}$ Irlanda, em $1^{\circ}$ de janeiro de 2019, surgiu a primeira UT, a Technological University Dublin. A instituição, segunda maior do país e detentora de uma história iniciada no ano de 1887, é o resultado do amalgamado de três institutos de tecnologia: o Dublin Institute of Technology, o Institute of Technology Tallaght e o Institute of 
Technology Blanchardstown. Outras duas UTs, originadas da fusão de institutos de tecnologia, estão em constituição: a Munster Technological University e a Technological University of the South East (Houghton, 2020).

A transformação é fruto de uma estratégia para o ensino superior irlandês. Na estratégia, uma UT é formada pela junção de institutos tecnológicas e tem como foco a preparação para funções profissionais complexas em um mundo tecnológico em mudança. O profissional formado pelas UTs tem como papel disseminar o conhecimento de forma a atender às necessidades da sociedade e das empresas, com especial atenção para as demandas da região em que a universidade está localizada (Irish, 2018).

Houghton (2020), com foco nos onze institutos de tecnologia restantes na Irlanda, para compreender as implicações da transformação por aglutinação, examinou estratégia similar colocada em prática no Reino Unido em 1992. É mister destacar que outros países, como a Austrália (Hatton, 2002; Mildred, 2002) e a Noruega (Kyvik, 2002; Norgård e Skodvin, 2002) vivenciaram processos aglutinativos equivalentes. No estudo, Houghton (2020) classifica o processo como um rompimento fundamental entre os institutos politécnicos e as universidades irlandesas. O efeito prático do processo foi uma espécie de corrida para a realização de coalizões e, o prêmio final, a concessão da condição de UT (Harkin e Hazelkorn, 2015).

O ponto de partida de Houghton (2020) é a experiência do vizinho mais próximo da Irlanda, o Reino Unido, que apresentava um sistema binário equivalente e transformou seus politécnicos em universidades. Para Pratt (1997), o fato dos politécnicos se autodenominarem universidades ocultou que estas universidades se tornaram politécnicos. A experiência é classificada como uma derivação vocacional (Doern, 2008). Para Houghton (2020) existe uma ameaça real de perda vocacional dos institutos politécnicos com a transformação em universidade.

Cunnane (2018) teme que, no processo de transformação, as novas instituições se desenvolvam com um $\mathbf{U}$ maiúsculo e um $\mathbf{t}$ minúsculo para aparecerem nos rankings mundiais. A busca pela inserção em rankings mundiais supõe a orientação para critérios distintos daqueles que sempre fizeram os institutos politécnicos irlandeses ao atenderem muito bem suas comunidades.

No Reino Unido, os politécnicos ao se tornarem UT, perderam muito de sua orientação vocacional e se tornaram vítimas da derivação vocacional que busca emular as universidades tradicionais em termos de disciplinas e de orientação (Houghton, 2020). As universidades transformadas tornaram-se, por vezes, imitações bastante pálidas desse tipo de instituição, sem filosofia e propósito coerentes.

A guisa de comparação, Houghton (2020) enxerga uma distinção importante e lamentável entre o que está ocorrendo na Irlanda em relação ao que ocorreu no Reino Unido. No Reino Unido, os politécnicos transformados adotaram a nomenclatura de universidade. Na Irlanda, tal qual ocorreu em outros países europeus como a Holanda e a Finlândia, será exigido o uso da nomenclatura UT para os institutos politécnicos que se tornarem universidades. Com efeito, em um sistema sem diferenciação, pode-se criar um agrupamento distinto de instituições de ensino superior (IES) em termos de status e, assim, reter de alguma forma o sistema binário existente (Houghton, 2020).

Na ótica de Houghton (2020), a nomenclatura universidade é relevante para a superação do status inadequado e desatualizado de segunda classe que é frequentemente atribuído aos institutos politécnicos. Em comparação com as universidades, os institutos politécnicos, embora tenham estudantes de todo o espectro social, atendem desproporcionalmente os menos favorecidos (Irish, 2019). Não obstante, Houghton (2020) enxerga que, com o status de UT, os institutos podem se libertar de sua posição subserviente.

Segundo Houghton (2020), outros problemas perspectivados no processo irlandês em curso são:

a) conflitos decorrentes dos complexos processos de fusão; 
b) distanciamento espacial entre instituições fundidas e possível desmonte da política de clusters e regionalismo em curso no ensino superior irlandês;

c) desenvolvimento de uma cultura de pesquisa nas novas UTs que sempre tiveram foco no ensino e uma carreira sem incentivos para a pesquisa e para a meritocracia;

d) dificuldade de racionalizar o sistema com as fusões;

e) construção de uma nova cultura organizacional e de uma nova identidade com a vinda de sangue novo para a organização;

f) disputas políticas por posições de liderança.

Nesse cenário complexo e de incertezas, Houghton (2020), ao questionar o que será perdido na transição para o status de UT, revela preocupação com a manutenção do foco no local e no regional e na manutenção da missão diferenciada da nova instituição. A preocupação é alinhada com o medo de Cunnane (2018) do desenvolvimento de uma Ut (com t minúsculo) ou simplesmente universidade.

\section{O modelo da Universidade Tecnológica Federal do Paraná}

Com a transformação do Centro Federal de Educação Tecnológica do Paraná (CEFET-PR) na UTFPR, através da Lei n. ${ }^{\circ} 11.184$ (Brasil, 2005), o Brasil ganhou sua primeira universidade tecnológica.

A transformação gerou movimentos políticos importantes. Muitos CEFETs, localizados em todos os estados brasileiros, passaram a buscar a condição de UT (Pilatti, 2017). O movimento era mais robusto nos CEFETs do Rio de Janeiro e de Minas Gerais. Estes CEFETs apresentavam indicadores acadêmicos similares aos do Centro transformado.

Na tentativa de evitar um desmonte do sistema federal voltado à formação técnica de nível médio, o mais importante do país, com a transposição para o nível superior, o Ministério da Educação (MEC) editou o Decreto n. ${ }^{\circ} 6.905$ (Brasil, 2007). O Decreto facultava a transformação dos CEFETs em Institutos Federais de Educação, Ciência e Tecnologia (IFs). A condição de IF proporcionava ganhos para os CEFETs e limites para evitar o deslocamento para o nível superior. O CEFET-MG e o CEFET-RJ não aderiram ao decreto (Ciavatta, 2006) e, depois de mais de uma década, continuam buscando a condição de UT (Pilatti, 2017). O impedimento revela óbice ao modelo por parte de governos com orientações políticas distintas.

Transformada, em 2008, a UTFPR aderiu ao Programa de Apoio a Planos de Reestruturação e Expansão das Universidades Federais (Reuni). O Programa possibilitou a UTFPR ampliar o processo de interiorização e a oferta majoritária de cursos engenharias. Com a expansão, o perfil do quadro docente foi deslocado do magistério do ensino básico, técnico e tecnológico (EBTT) para o magistério superior (MS) e se tornou mais acadêmico. A característica mais importante do CEFET-PR e de uma UT, a proximidade com o setor produtivo, com o perfil acadêmico dos docentes está se perdendo. Em média, os docentes do CEFET-PR apresentavam experiência profissional significativa e baixos índices de qualificação docente. Com a transposição, está ocorrendo uma inversão importante deste quadro (Pilatti, 2017).

Com o Reuni, a UTFPR transformou-se em uma das dez maiores universidades federais do Brasil. Constituída por campi em 13 cidades do Estado do Paraná, a UTFPR é, entre todas as universidades federais brasileiras, a com maior número de campus fora de sede. Nos campi do interior estão matriculados aproximadamente $70 \%$ dos alunos da instituição. Com o Programa Reuni, a UTFPR interiorizou os cursos de engenharia, até então restritos a sede em Curitiba. Com os novos cursos, entre as universidades públicas, a UTFPR passou a ser a instituição que mais oferta vagas de engenharia no Brasil. A oferta anual, em média, é o dobro da segunda instituição que mais oferta vagas, a Universidade Federal de Santa Catarina (UFSC). No processo de migração para as engenharias, ocorreu o fechamento de cursos superiores de tecnologia e técnicos e um crescimento importante na pós- 
graduação. Estes são os movimentos mais notórios da instituição transformada e permitem uma ideia parcial do que é uma UT no Brasil.

Avançando na resposta da pergunta o que é uma UT no Brasil? é bastante factível utilizar contornos tracejados na Lei de criação da UTFPR (Brasil, 2005). Para tal, quatro artigos da Lei são relevantes. O primeiro deles é o art. $2^{\circ}$. No artigo são apresentados os princípios da UTFPR:

I- $\quad$ ênfase na formação de recursos humanos, no âmbito da educação tecnológica, nos diferentes níveis e modalidades de ensino, para os diversos setores da economia, envolvidos nas práticas tecnológicas e na vivência com os problemas reais da sociedade, voltados, notadamente, para o desenvolvimento socioeconômico local e regional;

II - valorização de lideranças, estimulando a promoção social e a formação de cidadãos com espírito crítico e empreendedor;

III - vinculação estreita com a tecnologia, destinada à construção da cidadania, da democracia e da vida ativa de criação e produção solidárias;

IV - desenvolvimento de cultura que estimule as funções do pensar e do fazer, associando-as às atividades de ensino, pesquisa e extensão;

V- integração da geração, disseminação e utilização do conhecimento para estimular o desenvolvimento socioeconômico local e regional;

VI - aproximação dos avanços científicos e tecnológicos com o cidadão-trabalhador, para enfrentar a realidade socioeconômica em que se encontra;

VII - organização descentralizada mediante a possibilidade de implantação de diversos campi, inserindose na realidade regional, oferecendo suas contribuições e serviços resultantes do trabalho de ensino, da pesquisa aplicada e extensão;

VIII - articulação e integração verticalizada entre os diferentes níveis e modalidades de ensino e integração horizontal com o setor produtivo e os segmentos sociais, promovendo oportunidades para a educação continuada;

IX - organização dinâmica e flexível, com enfoque interdisciplinar, privilegiando o diálogo permanente com a realidade local e regional, sem abdicar dos aprofundamentos científicos e tecnológicos; e

$X$ - maximização quanto ao aproveitamento dos recursos humanos e uso da infraestrutura existente pelos diferentes níveis e modalidades de ensino.

(Brasil, 2005).

No art. 3으, as finalidades:

I- desenvolver a educação tecnológica, entendida como uma dimensão essencial que ultrapassa as aplicações técnicas, interpretando a tecnologia como processo educativo e investigativo para gerála e adaptá-la às peculiaridades regionais;

II - aplicar a tecnologia compreendida como ciência do trabalho produtivo e o trabalho como categoria de saber e produção; e

III - pesquisar soluções tecnológicas e desenvolver mecanismos de gestão da tecnologia, visando a identificar alternativas inovadoras para resoluções de problemas sociais nos âmbitos local e regional.

(Brasil, 2005).

No art. 4으, os objetivos:

I- $\quad$ ministrar em nível de educação superior:

a) cursos de graduação e pós-graduação, visando à formação de profissionais para as diferentes áreas da educação tecnológica; e 
b) cursos de licenciatura, bem como programas especiais de formação pedagógica, com vistas à formação de professores e especialistas para as disciplinas nos vários níveis e modalidades de ensino de acordo com as demandas de âmbito local e regional;

II - ministrar cursos técnicos prioritariamente integrados ao ensino médio, visando à formação de cidadãos tecnicamente capacitados, verificadas as demandas de âmbito local e regional;

III - oferecer educação continuada, por diferentes mecanismos, objetivando a capacitação, o aperfeiçoamento, a especialização e a atualização de profissionais, em todos os níveis de ensino, nas áreas da educação tecnológica;

IV - realizar pesquisas, estimulando atividades criadoras e estendendo seus benefícios à comunidade, promovendo desenvolvimento tecnológico, social, econômico, cultural, político, ambiental; e

V - desenvolver atividades de extensão de acordo com os princípios e finalidades da educação tecnológica, em articulação com o setor produtivo e os segmentos sociais.

(Brasil, 2005).

E no art. 5, onde consta: "A UTFPR, observado o princípio de indissociabilidade entre o ensino, pesquisa aplicada e extensão, organizará sua estrutura e forma de funcionamento, nos termos desta Lei e das normas legais pertinentes" (Brasil, 2005).

Os contornos presentes, em alguma medida, produzidos por desdobramentos advindos do MEC e internos, apontam para um deslocamento do eixo estruturante proposto na Lei de criação da UTFPR.

Outro elemento possível de ser considerado no estabelecimento do que é uma UT no Brasil é o Projeto PolíticoPedagógico Institucional (PPI) da Instituição (Universidade Tecnológica Federal do Paraná, 2007). O projeto de futuro da UTFPR recebeu forte influência do modelo francês. A influência pode ser explicada em função de, historicamente, o CEFET-PR ter importantes parcerias com as UTs francesas. É identificável, também, no PPI a presença de alguma influência do modelo alemão (Universidade Tecnológica Federal do Paraná, 2007). 0 capítulo 7 - A identidade da UTFPR, parte mais conceitual e filosófica do documento, é organizado em cinco partes. As duas primeiras são uma espécie de detalhamento da Lei $n .^{\circ} 11.184$ (Brasil, 2005). Na terceira parte, são discutidos os mecanismos de interação com a comunidade externa. O desenvolvimento da comunidade externa e a gestão democrática são, respectivamente, as duas últimas partes do capítulo.

No documento evidencia-se a preocupação da UTFPR preencher uma lacuna no processo relacional universidade-empresa, produzido pelo distanciamento real destes atores sociais. Este distanciamento é, em parcela importante, determinado pelo utilitarismo de esquerda presente nas universidades públicas brasileiras (Brito Cruz, 2010). A Figura 1 perspectiva uma zona imaginária na qual a UTFPR projetou sua atuação.

Figura 1

Concepção da UTFPR

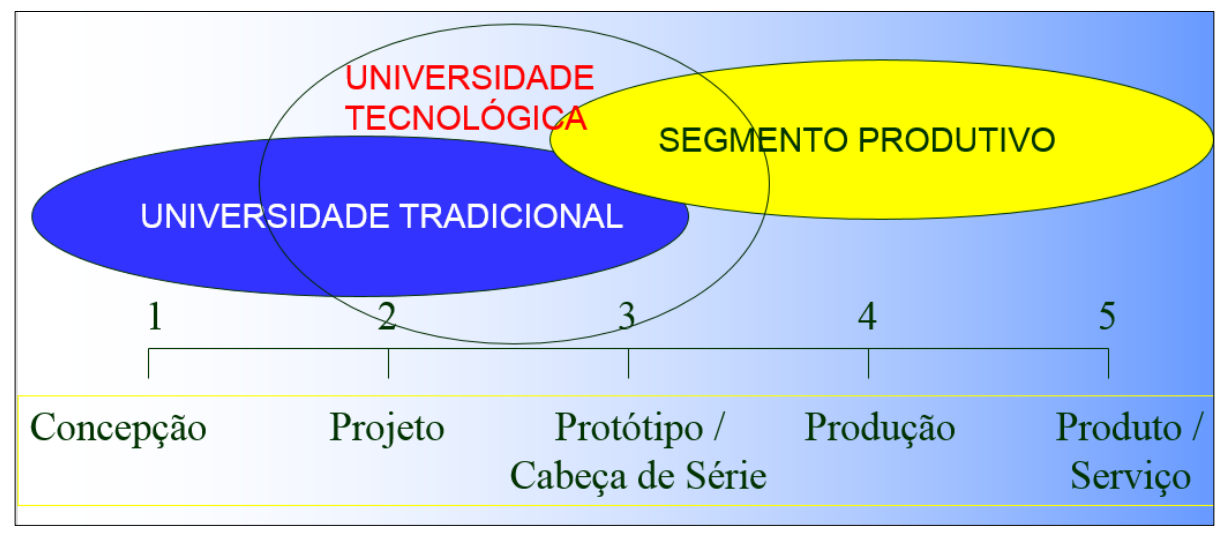

Fonte: Universidade Tecnológica Federal do Paraná (2007). 
O distanciamento pode, em medida importante, ser explicado pela pesquisa. No Brasil a pesquisa básica acontece na universidade e o desenvolvimento necessário à inovação e à competitividade nas empresas. Estar mais próximo do setor produtivo implica no deslocamento da pesquisa básica para a pesquisa aplicada. Com efeito, tem-se uma aproximação singular com a ideia de educação tecnológica (Figura 2).

Figura 2

Eixos do sistema educacional

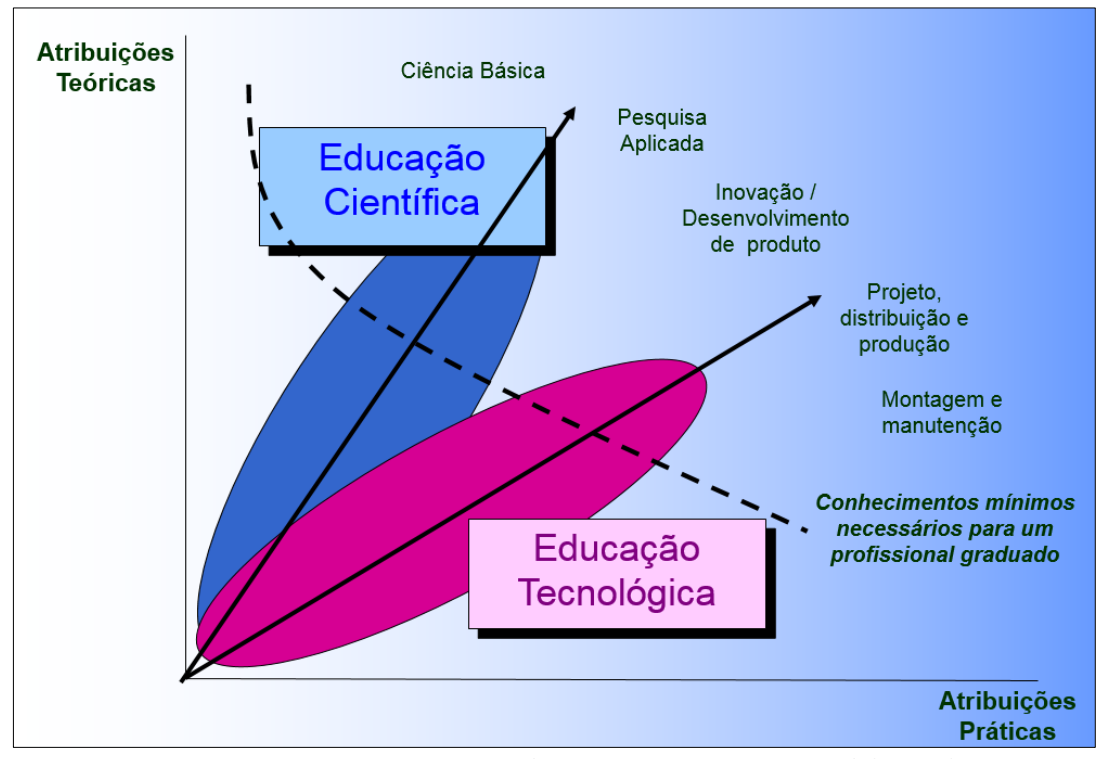

Fonte: Universidade Tecnológica Federal do Paraná (2007).

A prospecção dos papeis das diferentes instituições de ensino estão representados na Figura 3.

Figura 3

Atribuições no sistema educacional brasileiro

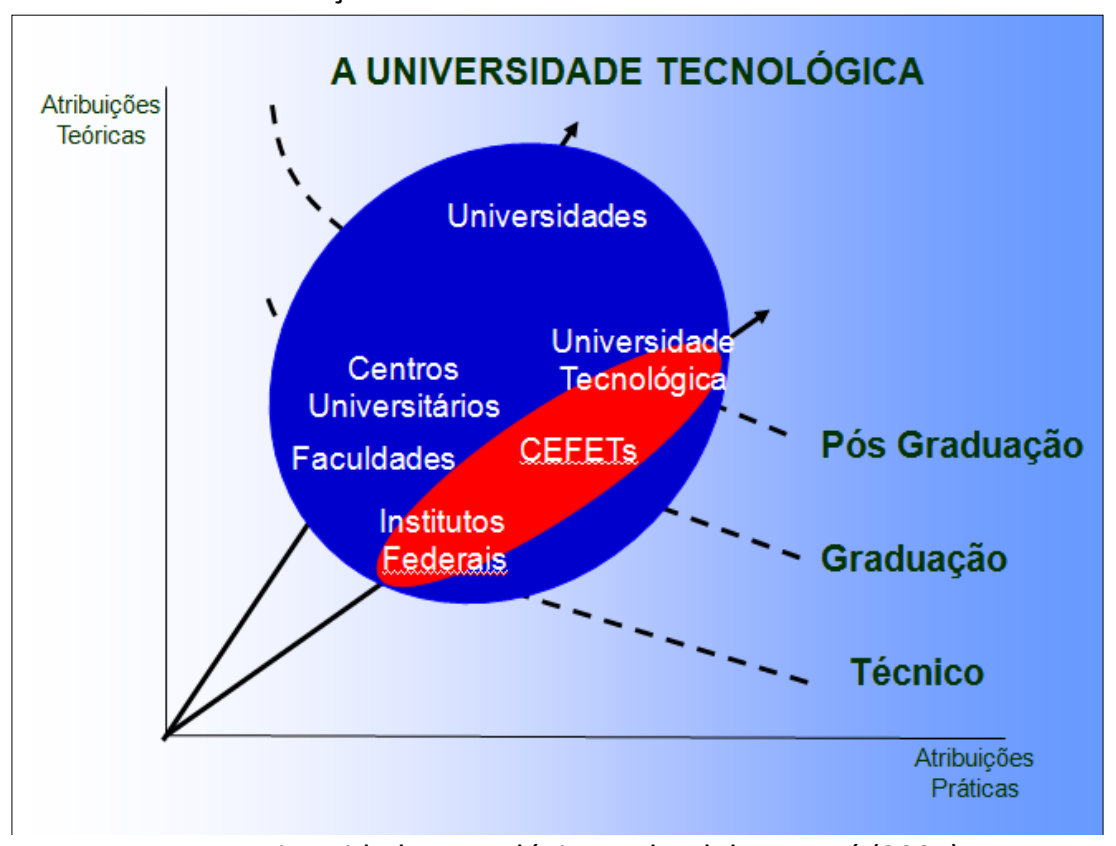

Fonte: Universidade Tecnológica Federal do Paraná (2007).

Na concepção da UTFPR, a ideia de extensão, presente na indissociabilidade preconizada pela Constituição Cidadã, é particularizada para a extensão tecnológica e produção, uma das oito áreas temáticas da extensão (Fórum de Pró-Reitores das Instituições Públicas de Educação Superior Brasileiras, 2012). A procriação se efetivou 
com a institucionalização da Pró-Reitoria de Relações Empresariais e Comunitárias (PROREC). Nenhuma universidade brasileira tem uma pró-reitoria análoga.

Na junção dos dois documentos examinados, a Lei de criação e no primeiro PPI, tem-se os contornos do UT do Brasil. A inexistência de modelo anterior e a recentidade da UTFPR permitem perspectivar o modelo como em construção. Conquanto, o modelo adotado na UTFPR apresenta alguns limites que permitem distinções das universidades clássicas e se aproxima da ideia de UT internacionalmente aceita. Entre os limites têm-se, a ênfase na educação tecnológica, o foco em problemas reais, a perspectiva do desenvolvimento socioeconômico local e regional, a orientação para a pesquisa aplicada e para a articulação com o setor produtivo. 0 desvio das propostas iniciais encontra-se no distanciamento institucional dos cursos técnicos que tendem ao desaparecimento. Em termos práticos, diferente do tripé ensino, pesquisa e extensão que serve de fundamento para a ideia de universidade no Brasil, a proposta é edificada no tripé educação tecnológica, pesquisa aplicada e relacionamento empresarial. Na questão do relacionamento empresarial, na França, referência utilizada pela UTFPR, a questão é colocada em termos de transferência de tecnologia (Cechin, 2019; Lievore e Pilatti, 2018).

Do ponto de vista legal, nada distingue a UTFPR das demais universidades federais, ou seja, não existe um sistema binário no Brasil. A inexistência de distinções coloca para a UTFPR problemas legais, como a possibilidade muito limitada de ter profissionais da indústria em seus quadros, para a efetivação da ideia de UT, mais prática e próxima do setor produtivo, uma universidade do mundo real. Nem mesmo legislações que projetaram avanços importantes, como a Lei da Inovação, produziram possibilidades reais para um cenário efetivamente voltado para a tecnologia. É adequado destacar que as distinções existem quando se examina, por exemplo, as universidades e os IFs, mas são distinções de instituições com atuação em graus distintos de ensino.

Tendo em vista que a linha que separa o clássico do tecnológico é tênue, o deslocamento pendular, principalmente do tecnológico para o tradicional, é bastante factível. Nos termos de Cunnane (2018) é a Ut escrevendo seu t minúsculo. A aproximação do modelo clássico é uma preocupação revelada pela UTFPR nos seus projetos pedagógicos (Universidade Tecnológica Federal do Paraná, 2014, 2019).

Estudos como os de Lievore e Pilatti (2018) e Costa e outros (2019) mostram o afastamento do modelo constitutivo. Chama atenção, também, a política e a definição de diretrizes para criação de cursos regulares presenciais vinculados à Pró-Reitoria de Graduação e Educação Profissional, aprovada no órgão máximo da Instituição (Universidade Tecnológica Federal do Paraná, 2018). O documento é uma tentativa clara de correção de curso. Evidentemente é possível pensar numa instituição que usa a tecnologia como meio, mas nem sempre a prática corresponde com o projetado. A inexistência de referências torna a manutenção do foco bastante complexa. Iniciativas presentes na progênie da UTFPR, como a disciplina de empreendedorismo em todos os cursos, se perderam. A ideia de cursos práticos também se perdeu. A comparação de cursos ofertados na UTFPR com os ofertados em universidades clássicas, quase sempre, apresenta muita semelhança.

Uma explicação possível, encontra-se na percepção de que as UTs são instituições de segunda classe e destinadas às classes mais desfavorecidas (Houghton, 2020). Em oposição, a ideia de universidade, revestida de uma tradição quase milenar, é detentora de um status superior. Para Lessa (2005), "[...] a Universidade não é o andaime da educação nacional, e sim seu alicerce. [...]. É o modo civilizado de presença, em vez da baioneta e da moeda dominante" (pp. A18). A percepção, em alguma medida, explica o trânsito das escolas da mão na graxa para o nível de ensino superior do país.

Atualmente, tramita na Câmara dos Deputados o Projeto de Lei n ${ }^{\circ} 1.453$ (Brasil, 2021). 0 projeto, em essência, propõe alterar a Lei $n^{\circ} 11.892$ (Brasil, 2008). Na proposta, destaque para o art. 8 que eleva do mínimo de 50 para $75 \%$ da oferta de vagas em cursos de educação profissional técnica de nível médio. Na proposta, a UTFPR é retirada da rede. $O$ projeto de lei pode ser interpretado como uma tentativa de retorno do pêndulo da rede para 
o nível médio. Em termos práticos, se o projeto de lei for aprovado nos termos propostos, as condições técnicas para a criação de novas UTs no Brasil ficam diminuídas.

\section{Conclusões}

Em média, o forte relacionamento com o mercado e as particularidades nas missões universitárias produzem a ideia de UT. Na primeira missão, o ensino é vislumbrado como mais prático que nas universidades clássicas $\mathrm{A}$ predominância da pesquisa aplicada é a especialização da segunda missão e alinhada com o desenvolvimento de tecnologia. Na terceira missão, o escopo está na transferência de tecnologia orientada pelo modelo market-pull (pesquisa orientada pelas necessidades de mercado).

Como representação, pode-se alocar as UTs mais próximas do extremo especializado, mesmo quando a UT usa a tecnologia como meio. Em países com um sistema binário do ensino superior claramente estabelecido, a representação mental proposta encontra forte congruência com a realidade concreta. Enquanto um modelo difundido principalmente no século XX, em comparação com as universidades clássicas, o modelo de UT ainda padece da tradição e dos contornos mais precisos, além de tender, em muitos casos, para universalidade.

No caso brasileiro, o modelo é representado pelos contornos da única instituição caracterizada como UT, a UTFPR. No curtíssimo percurso desta Instituição é evidente o crescente distanciamento entre o modelo concebido e a prática institucional. Em parte, a inexistência de um sistema binário explica a situação. Elementos como falta de tradição, desconhecimento do modelo, sistemas avaliativos do ensino superior brasileiro, limitações legais, encaminhamentos políticos e simbolismo, complementam de forma importante a resposta. Ademais, é evidente que, diferente do que ocorre em muitos países desenvolvidos e em desenvolvimento, o modelo de UT não compõe o ideário de políticas para o devir educacional brasileiro.

\section{Referências bibliográficas}

Brasil. (2007). Decreto $n^{\circ} 6.095$, de 24 de abril de 2007. Estabelece diretrizes para o processo de integração de instituições federais de educação tecnológica, para fins de constituição dos Institutos Federais de Educação, Ciência e Tecnologia - IFET, no âmbito da Rede Federal de Educação Tecnológica. Diário Oficial da União: seção 1, Brasília, DF, n. 79, pp. 6-7, 25 abr. Disponível em:

https://pesquisa.in.gov.br/imprensa/jsp/visualiza/index.jsp?jornal=1\&pagina=6\&data=25/04/2007.

Brasil. (2005). Lei no 11.184, de 7 de outubro de 2005. Dispõe sobre a transformação do Centro Federal de Educação Tecnológica do Paraná em Universidade Tecnológica Federal do Paraná e dá outras providências. Diário Oficial da União: seção 1, Brasília, DF, n. 195, pp. 1-2, 10 out. Disponível em: https://pesquisa.in.gov.br/imprensa/jsp/visualiza/index.jsp?jornal=1\&pagina=1\&data=10/10/2005.

Brasil. (2008). Lei no 11.892, de 29 de dezembro de 2008. Institui a Rede Federal de Educação Profissional, Científica e Tecnológica, cria os Institutos Federais de Educação, Ciência e Tecnologia, e dá outras providências. Diário Oficial da União: seção 1, Brasília, DF, n. 253, pp. 1-3, 30 dez. Disponível em: https://pesquisa.in.gov.br/imprensa/jsp/visualiza/index.jsp?jornal=1\&pagina=1\&data=30/12/2008.

Brasil. (2021). Projeto de Lei $n^{\circ}$ 1.453/2021. Altera a Lei $n=11.892$, de 28 de dezembro de 2008, que institui a Rede Federal de Educação Profissional, Científica e Tecnológica, cria os Institutos Federais de Educação, Ciência e Tecnologia, e dá outras providências, para expandir a inovação e o alcance dos cursos técnicos, promover estratégias para a profissionalização e estimular o emprego. Câmara dos Deputados, Brasília, DF. Disponível em: https://www.camara.leg.br/propostas-legislativas/2278542. 
Brito Cruz, C. H. (2010). Ciência, tecnologia e inovação no Brasil: desafios para o período 2011 a 2015. Interesse Nacional. Disponível em: https://www.ifi.unicamp.br/ brito/artigos/CTI-desafios-InteresseNacional07082010-FINAL.pdf.

Castells, M. (2005). A sociedade em rede. São Paulo: Paz e terra.

Cechin, M. R. (2019). Estudo comparativo entre a Universidade Tecnológica Federal do Paraná e as universidades de Tecnologia da França. Tese (Doutorado em Ensino de Ciência e Tecnologia) Universidade Tecnológica Federal do Paraná, Ponta Grossa. Disponível em: http://repositorio.utfpr.edu.br/jspui/handle/1/4041.

Cechin, M. R., Pilatti, L. A. e Ramond, B. (2021). Maio de 68: contribuições para nascer a primeira universidade de tecnologia na França. Cadernos de História da Educação, 20(e013). DOI: https://doi.org/10.14393/chev20-2021-13.

Ciavatta, M. (2006). Os centros federais de educação tecnológica e o ensino superior: duas lógicas em confronto. Educação \& Sociedade, 27(96), 911-934. DOI: https://doi.org/10.1590/S010173302006000300013.

Costa, A. da, Pilatti, L. A., Santos, C. B. dos e Lievore, C. (2019). Perfil dos docentes de jovens universidades brasileiras: estudo comparativo entre UTFPR e UFABC. Revista Brasileira de Ensino de Ciência e Tecnologia, 12(1), 523-538. DOI: http://dx.doi.org/10.3895/rbect.v12n1.9575.

Cunnane, V. (2018). Technological universities should bring out the best of both sectors. The University Times. Disponível em: http://www.universitytimes.ie/2018/09/technological-universities-should-bring-out-thebest-of-both-sectors/.

Doern, B. (2008). "Polytechnics" in higher education systems: a comparative review and policy implications for Ontario. Toronto: The Higher Education Quality Council of Ontario. Disponível em: https://heqco.ca/wpcontent/uploads/2020/03/Polytechnics-in-Higher-Education-Systems_A-Comparative-Review-and-PolicyImplications-for-Ontario.pdf.

Du Pré, R. (2010). Universities of technology in the context of the South African higher education landscape. In: SOUTH AFRICAN COUNCIL ON HIGHER EDUCATION (CHE). Kagisano $n^{\circ}$ 7: Universities of technology: deepening the debate (pp. 1-41). Pretoria: CHE. Disponível em: https://cms.cut.ac.za/Files/Froala/bb2e79f0-7d35-4112-9a0a-6afcb8b5200c.pdf.

Fórum de Pró-Reitores de Extensão das Universidades Públicas Brasileiras. (2012). Política Nacional de Extensão Universitária. Manaus: FORPROEX. Disponível em: https://proex.ufsc.br/files/2016/04/Pol\%C3\%ADtica-Nacional-de-Extens\%C3\%A3o-Universit\%C3\%A1ria-ebook.pdf.

France. (1968). Loi n68-978 du 12 novembre 1968 d'orientation de l'enseignement supérieur. Paris: République Française. Disponível em: https://www.legifrance.gouv.fr/loda/id/JORFTEXT000000693185/2000-0621/?isSuggest=true.

Harkin, S.; Hazelkorn, E. (2015). Institutional mergers in Ireland. In: CURAJ, A. e outros. (ed.). Mergers and alliances in higher education: international practice and emerging opportunities (pp. 105-121). Dordrecht, Netherlands: Springer. 
Hasanefendic, S. (2018). Responding to new policy demands: a comparative study of Portuguese and Dutch non-university higher education organizations. Thesis (Doctorate in Public Policy) - Instituto Universitário de Lisboa, Lisboa. Disponível em: https://repositorio.iscte-iul.pt/handle/10071/16590.

Hatton, E. J. (2002). Charles Sturt University: a case study of institutional amalgamation. Higher Education, 44(1), 5-27. DOI: https://doi.org/10.1023/A:1015504909483.

Hayhoe, R. (1989). China's universities and Western academic models. Higher Education, 18, 49-85. DOI: https://doi.org/10.1007/BF00138961.

Helmann, C. L. (2019). Universidade Tecnológica Federal do Paraná e Instituto Politécnico de Bragança: um estudo comparativo. Tese (Doutorado em Ensino de Ciência e Tecnologia) - Universidade Tecnológica Federal do Paraná, Ponta Grossa. Disponível em:

http://repositorio.utfpr.edu.br:8080/jspui/handle/1/4148.

Houghton, F. (2020). Technological universities in Ireland: the new imperative. Irish Journal of Academic Practice, 8(1). Disponível em: https://arrow.tudublin.ie/ijap/vol8/iss1/12/.

Irish. (2019). Department of Education. Higher Education Authority (HEA). A spatial \& socio-economic profile of higher education institutions in Ireland. Dublin: Higher Education Authority. Disponível em:

https://hea.ie/assets/uploads/2019/10/Higher-Education-Spatial-Socio-Economic-Profile-Oct-2019.pdf.

Irish. (2018). Department of Education. Higher Education Authority (HEA). Process and Criteria for Designation as a Technological University. Disponível em: https://www.education.ie/en/The-Education-System/HigherEducation/Process-and-Criteria-for-Designation-as-a-Technological-University.pdf.

Kyvik, S. (2002). The merger of non-university colleges in Norway. Higher Education, 44, 53-72. DOI: https://doi.org/10.1023/A:1015561027230.

Laya, M. S. (2009). Technological universities: a relevant educational model for Mexico? In: RABY, R. L.; VALEAU, E. J. (ed.). Community College Models (pp. 219-233). Dordrecht: Springer. DOI: https://doi.org/10.1007/978-1-4020-9477-4_13.

Lessa, C. (2005). Universidade pública e nação. Jornal do Brasil, Rio de Janeiro, Economia \& Negócios, A18. Disponível em: https://www2.senado.leg.br/bdsf/bitstream/handle/id/64742/noticia.htm?sequence=1\&isAllowed=y.

Lewis, M. S. (1991). The polytechnics: a peculiarly British phenomenon. Metropolitan Universities, 2(4), 24-34. Disponível em: https://journals.iupui.edu/index.php/muj/article/view/19224.

Lievore, C.; Pilatti, L. A. (2018). Entre o tecnológico e o clássico: o modelo de universidade da UTFPR. Trabalho \& Educação, 27(1), 135-159. Disponível em: https://periodicos.ufmg.br/index.php/trabedu/article/view/9725.

Lievore, C.; Pilatti, L. A.; Teixeira, J. A. S. (2021a). Shaping for the future: professionalizing higher education and implications on the scientific policies of Brazil and Portugal. SN Social Sciences, 1. DOI: https://doi.org/10.1007/s43545-020-00019-z.

Lievore, C.; Pilatti, L. A.; Teixeira, J. A. S. (2021b). Universities of applied sciences in Brazil and in Portugal from conception to practice. Interchange, 52, 115-132. DOI: https://doi.org/10.1007/s10780-020-09412-2.

McKenna, S.; Sutherland, L. (2006). Balancing knowledge construction and skills training in universities of technology. Perspectivies in Education, 24, 15-24. Disponível em: 
https://www.semanticscholar.org/paper/Balancing-knowledge-construction-and-skills-in-of-MckennaSutherland/e34524bcc30462cb464d646b23c3d9118cea0823.

Mildred, G. (2002). Launching the Unified National System: what happened in South Australia. Higher Education, 44, 29-51. Disponível em: https://link.springer.com/article/10.1023/A:1015556910392.

Norgård, J. D.; Skodvin, O.-J. (2002). The importance of geography and culture in mergers: a Norwegian institutional case study. Higher Education, 44, 73-90. Disponível em: https://link.springer.com/article/10.1023/A:1015513111300.

Perez-Esparrells, C.; Orduna-Malea, E. (2018). Do the technical universities exhibit distinct behaviour in global university rankings? A Times Higher Education (THE) case study. Journal of Engineering and Technology Management, 48, 97-108. DOI: https://doi.org/10.1016/j.jengtecman.2018.04.007.

Pilatti, L. A. (2017). Internalização da interdisciplinaridade como condição para a internacionalização da Universidade Tecnológica Federal do Paraná - UTFPR. In: PHILIPPI JR, A.; FERNANDES, V.; PACHECO, R. C. S. (org.). Ensino, pesquisa e inovação: desenvolvendo a interdisciplinaridade (pp. 102-119). Barueri: Manole.

Pilatti, L. A.; Lievore, C. (2018). Redes de universidades: o caso da RUTyP. Educación Superior y Sociedad, 28(28), 127-154. Disponível em: https://www.iesalc.unesco.org/ess/index.php/ess3/article/view/87.

Pratt, L. (1997). The polytechnic experiment: 1965-1992. Buckingham: Open University Press. Disponível em: https://archive.org/details/ERIC_ED415724.

Red de Universidades Tecnológicas y Politécnicas da América Latina y El Caribe. (2017). Ejes estratégicos. Disponível em: https://www.redutyp.org/.

Universidade Tecnológica Federal do Paraná. (2014). Plano de Desenvolvimento Institucional: 2013-2017. Curitiba: UTFPR. Disponível em: http://portal.utfpr.edu.br/documentos/reitoria/documentosinstitucionais/pdi/pdi_2013-2017.

Universidade Tecnológica Federal do Paraná. (2018). Política e definição de diretrizes para criação de cursos regulares presenciais vinculados a Pró-Reitoria de Graduação e Educação Profissional: PROGRAD/UTFPR. Curitiba: UTFPR. Disponível em: https://cloud.utfpr.edu.br/index.php/s/DoCD8aBfVN6tsfl.

Universidade Tecnológica Federal do Paraná. (2019). Projeto Pedagógico Institucional (PPI) da Universidade Tecnológica Federal do Paraná. Curitiba: UTFPR. Disponível em: https://cloud.utfpr.edu.br/index.php/s/Z3pqMqWkxbsCbLz.

Universidade Tecnológica Federal do Paraná. (2007). Projeto Político-Pedagógico Institucional (PPI). Curitiba: UTFPR. Disponível em: http://www2.td.utfpr.edu.br/eng_civil/pdf/ppi.pdf.

\section{(cc) BY-No}

Este trabalho está sob uma Licença Creative Commons Atribuição-NãoComercial 4.0 Internacional (CC BY-NC 4.0) 